\title{
EchoGéo
}

$58 \mid 2021$

Varia

\section{Dubaï et le sport, image d'une société fracturée}

\section{Raphaël Le Magoariec}

\section{(2) OpenEdition}

Journals

Édition électronique

URL : https://journals.openedition.org/echogeo/22590

DOI : 10.4000/echogeo.22590

ISSN : 1963-1197

\section{Éditeur}

Pôle de recherche pour l'organisation et la diffusion de l'information géographique (CNRS UMR 8586)

\section{Référence électronique}

Raphaël Le Magoariec, « Dubaï et le sport, image d'une société fracturée », EchoGéo [En ligne], 58 | 2021, mis en ligne le 31 décembre 2021, consulté le 12 février 2022. URL : http://

journals.openedition.org/echogeo/22590; DOI : https://doi.org/10.4000/echogeo.22590

Ce document a été généré automatiquement le 12 février 2022.

EchoGéo est mis à disposition selon les termes de la licence Creative Commons Attribution - Pas d'Utilisation Commerciale - Pas de Modification 4.0 International (CC BY-NC-ND) 


\title{
Dubaï et le sport, image d'une société fracturée
}

\author{
Raphaël Le Magoariec
}

\section{Introduction}

1 Sur le bord des pelouses du Sevens Stadium, en périphérie de Dubaï, sur la route d'AlAïn, c'est un samedi après-midi de fête. Le Emirates Airlines Dubaï Rugby Sevens réunit chaque année fin novembre sur ce complexe les meilleures sélections du rugby mondiales à sept. Sur les terrains annexes, ce tournoi rassemble, par ailleurs, des équipes de rugbymen et de rugbywomen amateurs expatriés résidant dans l'ensemble de la région du Golfe arabo-persique. Financé depuis 1996 par Emirates Airlines, ce tournoi est conçu comme un événement moteur dans la communication mondiale développée par l'émirat. Loin de l'affirmation de cette stratégie récente, la première mouture de cette compétition est lancée en 1970 par un groupe de travailleurs expatriés originaires de pays du Commonwealth. Ces amateurs de rugby, ayant obtenu un terrain de la part de l'émir Rachid Bin Mohammed Al-Maktoum en périphérie de la cité portuaire, à Al-Awir, souhaitaient élargir leur confrontation à des équipes d'expatriés autres que celle des militaires britanniques présents sur la base aérienne de Sharjah. En cet après-midi ensoleillé de cette fin du mois de novembre 2018, sur ces terrains annexes, les équipes qui s'affrontent apparaissent comme une survivance de cette genèse originelle du tournoi, loin de l'imaginaire développé par Dubaï à l'échelle mondiale. À travers l'organisation d'étapes de circuits sportifs mondiaux et d'une intense stratégie de sponsoring transnationale, prenant appui sur les sociétés de son «empire» du transport et sur l'intensification de sa politique de hub dans les années 1990, Dubaï accentue sur son territoire la mondialisation des espaces sportifs qui y émergent. L'extension à l'échelle mondiale de la politique de l'émirat, sous l'impulsion d'une nouvelle génération de décideurs menée par le prince héritier d'alors, Mohammed Bin Rachid Al-Maktoum, de même que son adaptation à la tertiarisation de l'économie mondiale, placent Dubaï dans la position de métropole motrice du Golfe 
arabo-persique. Dans ce mouvement que connaît l'émirat, le sport s'articule au sein de son territoire à travers différents systèmes.

2 Au fil des promenades, les études qualitatives menées au cœur de cet espace et l'observation par le regard du chercheur de ses différents terrains sportifs permettent de distinguer deux grandes sphères sportives, l'une horizontale, l'autre verticale. Ce premier constat, couplé à des entretiens menés avec plusieurs acteurs vivant à Dubaï ou y ayant vécu, émiriens ou expatriés, élabore les contours d'une plus large étude s'inscrivant dans le domaine de la géopolitique de l'émirat de Dubaï au regard du sport. Cet article se propose par conséquent d'étudier les systèmes organisationnels formels et / ou informels qui naissent au sein de son tissu sportif local. Il s'agit de partir de l'étude du concret de la pratique sportive, pour comprendre comment elle s'articule autour d'un hub, Dubaï, au caractère multiculturel et percevoir quels effets la métropolisation induit sur cet espace.

\section{L'émirat de Dubaï dans son espace régional, approche historique}

3 Émirat majeur de la fédération des Émirats arabes unis, Dubaï a construit sa stature par son histoire marchande, qui s'est développée au cours du $\mathrm{XX}^{\mathrm{e}}$ siècle, avant de s'appuyer dans la seconde moitié du $\mathrm{XX}^{e}$ siècle, sur ses minces ressources pétrolières pour accroître son rôle de hub, devenant un hub commercial mondial (Marchal, 2001; Davidson, 2008 ; Lavergne, 2009 ; Coates Ulrichsen, 2016). Rival historique d'Abu Dhabi, l'émirat de Dubaï s'est transformé en un pôle influent grâce à sa fonction de plateforme marchande et financière, tandis qu'Abu Dhabi s'est imposé comme acteur politique moteur de la fédération, grâce à ses ressources pétrolières conséquentes (Ulrichsen Coates, 2016). Il s'agit d'une industrie pétrolière diversifiée par l'investissement de l'émirat dans ses fonds souverains, Abu Dhabi Investment Authority et Mubadala, une force économique qui lui permet d'assoir sa domination sur les centres de décisions fédéraux (Ulrichsen Coates, 2016). Affaibli lors de la crise financière de 2008, proche d'une situation de banqueroute, l'émirat de Dubai trouve un soutien économique en l'émirat d'Abu Dhabi. En contrepartie de cette aide financière, une redéfinition géopolitique s'opère au sein de la fédération. À l'instar du Burj Khalifa, tour iconique de Dubaï, inaugurée en 2010, à laquelle est attribué en signe de reconnaissance à Abu Dhabi le nom de son émir Khalifa bin Zayed Al-Nahyan, ce contexte profite à l'émirat d'Abu Dhabi. Cette nouvelle conjoncture renforce Abu Dhabi qui assoit son leadership dans la définition de la politique fédérale, Dubaï se place désormais plus en retrait. L'émirat se concentre davantage sur ses investissements propres.

4 Autour de ces deux acteurs centraux, cinq émirats prennent part dans le nord du pays : Sharjah, Ajman, Umm Al-Quwaim, Ras Al-Khaimah, Fujairah, qui ne possèdent pas, ou très peu, de ressources pétrolières. Ces principautés vivent d'une économie centrée sur l'industrie, les transports et le commerce (Herb, 2014; Dumortier, 2013). La redistribution budgétaire se révèle être un outil politique central dans leur développement. Sur l'échiquier fédéral, ces émirats sont relégués à la marge de par leur profil économique. 


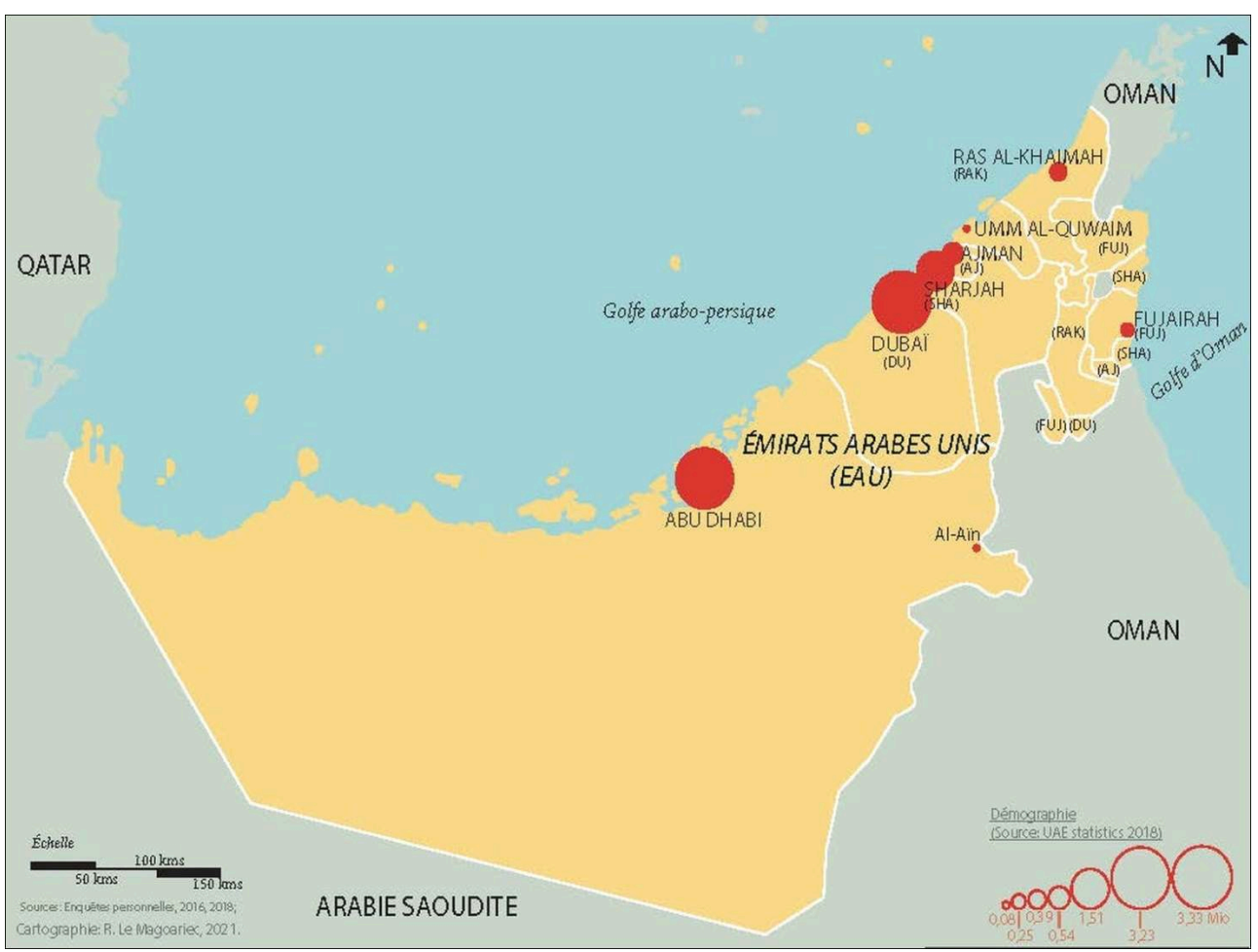

Auteur: R. Le Magoariec, 2021

Peu de productions scientifiques ont traité de Dubaï à travers le prisme du sport et encore moins en se basant sur un travail d'étude des espaces sportifs. L'investissement de l'émirat dans cette industrie est pris en exemple dans certains ouvrages traitant des EAU et de Dubaï sous l'angle du branding (Davidson, 2008; Coates Ulrichsen, 2017), de même des publications abordant le sujet du sport au Moyen-Orient de manière généraliste s'y intéressent en retenant ce même angle (Amara, 2012, 2019). Hormis l'anthropologue Souleyman Khalaf, qui a étudié de près le développement des courses de chameaux au sein des Émirats arabes unis, observant les grands camélodromes comme des lieux de "production de la culture nationale» (Khalaf, 2000), autour de grandes personnalités des familles, contrebalançant avec le caractère multiculturel des cités-émirats du pays(Khalaf, 1999, 2000), aucun travail n'a jusqu'ici abordé Dubaï uniquement à travers l'angle du sport. Revenir au territoire est capital pour comprendre qu'avant le sport comme outil de branding dans la construction de "l'imaginaire de la métropolisation» (Lussault, 2010), le sport possédait déjà un ancrage historique au sein de la société émirienne. L'intégration de sports britanniques, au premier rang desquels le football, est née essentiellement de l'interaction entre populations étrangères issues du Proche et Moyen-Orient avec les populations locales ; les marchands indiens présents à Dubaï ont également favorisé son essor. La cité portuaire et ses flux commerciaux ont été propices à l'éclosion de ces disciplines sportives sur cette partie du littoral de la fédération des Émirats arabes unis. Peu pris en considération et mal connu par les personnes étrangères aux réalités sportives des sociétés du Golfe arabo-persique, puisque ne correspondant pas aux codes des standards imposés par l'industrie du sport spectacle ${ }^{1}$, l'historicité sportive de ces sociétés n'intègre pas la communication mondiale déployée par Dubaï. Pourtant, elle reste aujourd'hui à la base de ce tissu sportif local et forme de plus les contours de la 
culture sportive émirienne. C'est à partir des clubs historiques de Dubaï, émanations de l'État-providence qui structurent le territoire dans les anciens quartiers de la cité portuaire, que nous allons commencer notre voyage au cœur des différentes strates sportives de l'émirat. Des quartiers historiques centraux bordant la crique, nous gagnerons ensuite des espaces du territoire périphériques encore peu investis par les autorités. Toutefois, sous l'impulsion de travailleurs étrangers subalternes, ces périphéries deviennent des lieux de pratiques sportives informelles centraux, au sein de ce tissu urbain, pour ces sportifs d'un jour, grands amateurs de cricket. Enfin, notre périple prendra fin autour des activités physiques des expatriés à hauts revenus et de leur besoin de trouver de possibles lieux de loisirs pour profiter de leur temps libre. Ainsi, notre parcours a pour but de nous amener au sein des différentes couches sportives qui prennent corps autour de ce hub mondial du Golfe arabo-persique, Dubaï.

Illustration 2 - Vue sur le Sevens Stadium lors du tournoi annuel de l'Emirates Dubaï Rugby Sevens (Dubaï, 2018)

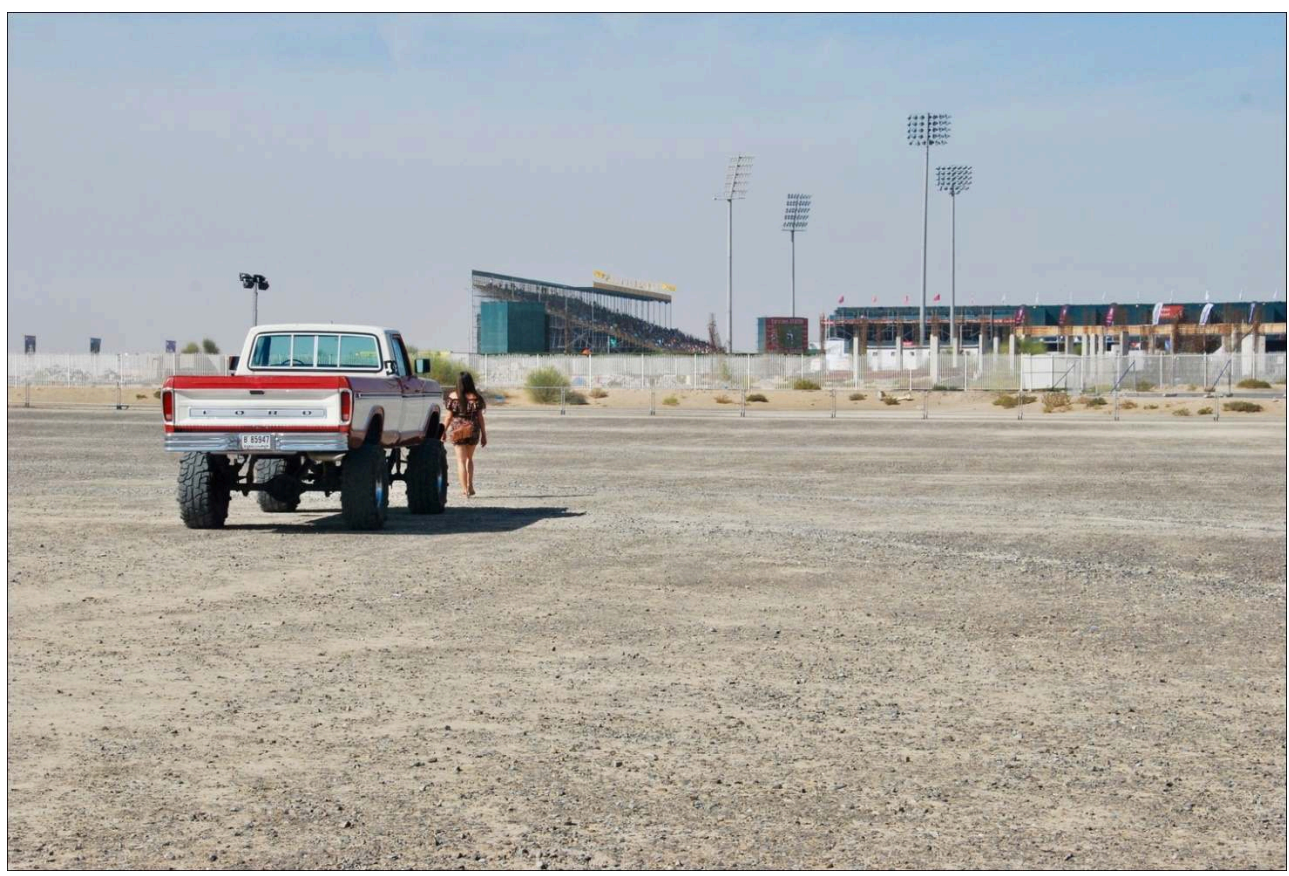

Auteur: R. Le Magoariec.

\section{L'espace sportif à Dubaï, reflets des fragmentations socio-spatiales}

6 À Dubaï, les lieux de pratique du sport laissent apparaître un espace fragmenté. Au travers de la «ville mondiale » qui prend corps à partir du milieu des années 1990 autour de cette cité portuaire en plein développement au cœur de la fédération des Émirats Arabes Unis, l'attractivité de la ville modifie son tissu sportif. La nouvelle phase migratoire ${ }^{2}$ concomitante à cet essor accentue la pratique de certains sports déjà intégrés à Dubaï lors des mouvements migratoires précédents. Le pôle de richesses qui se constitue autour de cette ville tout comme dans les autres émirats qui l'entourent, contribue à intégrer de nouvelles pratiques ou tendances sportives. Au cœur de cette ville et à l'échelle même de l'émirat, c'est une fragmentation socio-spatiale qui se dessine désormais dans les façons de vivre et de pratiquer le sport, que l'on soit acteur ou spectateur, expatrié ou golfien. Le sport contemporain se définit ainsi, dans un 
premier temps, par rapport à l'origine géographique des individus et, dans un deuxième temps, au regard du statut social que ceux-ci occupent au sein de cette société aux origines multiples.

\section{Au sein de leur société, quelle place pour les Émiriens dans le sport?}

\section{Un espace, produit de l'État-providence}

7 Le système sportif émirien s'est structuré, au cours des années 1970, financé par l'État providence à travers les clubs et fédérations qui en sont une émanation. Les autorités ont dès lors affiché la volonté de maintenir un espace sportif réservé aux citoyens de nationalité émirienne, et ce dès leur plus jeune âge. Minoritaires sur leur territoire national, les Émiriens ont ainsi un espace préservé leur permettant de s'adonner au sport proposé par les clubs gouvernementaux, en l'occurrence à Dubaï les clubs d'AlShebab, d'Al-Ahly ${ }^{3}$, d'Al-Nasr et d'Al-Wasl. Cette articulation participe au fonctionnement de l'État providence en raison de l'accès privilégié, sans frais d'inscription, garanti aux Émiriens souhaitant pratiquer une activité sportive. En plus de fonds distribués à partir du budget fédéral annuel, le sport est financé par les ministères du Sport ou par les gouvernements à l'échelle même des émirats. La situation varie d'un émirat à l'autre en fonction de sa situation économique. Possédant moins de ressources financières, les émirats du nord du pays, Ras Al-Khaimah, Umm AlQuwaim, Ajman et Fujairah, perçoivent essentiellement de l'argent par le biais des fédérations sportives nationales. Les fonds proviennent de l'administration fédérale en quête du développement des entités sportives gouvernementales de ces émirats ${ }^{4}$. À Dubaï, en plus de cette base financière fédérale, c'est le Dubaï Sports Council, à savoir le ministère du Sport de l'émirat, fondé en 2005, qui contribue chaque année au budget de ces clubs $^{5}$. L'accès au système sportif national est ainsi délimité par des normes juridiques qui se fondent sur la nationalité. La nationalité émirienne est la base juridique de l'accès aux différentes ligues fédérales, une limite qui varie en fonction des sports et de leur attractivité auprès du public émirien. Ce fonctionnement s'observe dans l'ensemble des pays du Conseil de Coopération du Golfe. Nonobstant cet accès privilégié, le sport émirien peine à être investi par les nationaux. Les enquêtés pointent en effet le manque, sur la durée, de "persévérance " ${ }^{6}$ et de présence de jeunes sportifs émiriens dans les clubs. Cela amène les institutions à ouvrir leurs portes à de jeunes sportifs issus de familles souvent originaires d'États dits "arabes », ou de pays du Golfe, vivant aux Émirats arabes unis depuis de nombreuses années. Par ce biais, il s'agit de garder des effectifs centrés sur le concept mouvant d'"arabité ", dans l'optique d'intégrer à l'avenir certains de ces joueurs aux sélections nationales. L'objectif est ainsi de préserver l'aspect identitaire, ce caractère reste toutefois assez opaque.

Plusieurs variables peuvent expliquer ce phénomène : d'une part, la faible démographie de la population émirienne estimée, en 2018 , au sein du pays à $11,4 \%$ des 9,5 millions d'habitants ${ }^{7}$ et à Dubaï à $7,5 \%$ des 3,3 millions d'habitants que compte l'émirat ${ }^{8}$; d'autre part, l'ensemble des loisirs qui composent aujourd'hui le tissu urbain dubaïote ; enfin, la conjoncture socio-économique induite par l'État-providence qui assure un certain confort matériel aux nationaux. Le sport, qui était présent et se développait au sein de la société émirienne, a ainsi été impacté par ces changements sociaux majeurs 
et n'apparaît pas comme un élément central. À ces trois phénomènes s'ajoute le fait que l'extension urbaine de Dubaï a éloigné les habitants émiriens de la crique autour de laquelle se sont développés et sont aujourd'hui situés les installations de ces clubs. Un éloignement qui décourage de nombreux parents à inscrire leurs enfants dans ces institutions. Pour tenter de supprimer ce désagrément, les clubs développent désormais dans des parcs à proximité des nouveaux lieux d'habitation des camps d'entrainement ${ }^{9}$. De plus, dans les écoles publiques émiriennes, l'éducation physique reste souvent délaissée par les instituteurs, une base qui n'encourage pas les enfants à persévérer dans un sport précis ${ }^{10}$. De même, les parents émiriens ne perçoivent pas souvent le sport comme une fin en soi, un sentiment qui résulte généralement de la non reconnaissance par le ministère du Travail émirien du statut de sportif professionnel ${ }^{11}$. Ce statut se décide à l'échelle même du club ou d'une ligue professionnelle et reste une question assez opaque. La carrière de sportif de haut niveau est dès lors peu prise au sérieux par de nombreux Émiriens. La pratique sportive, sous la forme d'éducation physique, ou du sport comme vecteur de loisir, ne s'inscrit pas uniquement dans une logique de performance et de professionnalisation, toutefois elle reste un socle essentiel dans la formation d'un vivier d'athlètes capables d'atteindre un niveau international. Pour tenter de rendre attractive la pratique du sport auprès de la jeunesse émirienne, les institutions émiriennes subventionnent des compétitions sportives dans les catégories de jeunes. C'est un fonctionnement propre au système d'État-providence qui est à l'œuvre. Par exemple, dans le domaine de la voile, les sommes d'argent attribuées aux vainqueurs de course ${ }^{12}$ peuvent très vite avoisiner les $4287 \mathrm{AED}^{13}$, une somme qui peut sembler démesurée pour des catégories de jeunes. Ce phénomène socio-économique tend majoritairement à susciter l'intérêt des enfants de familles reléguées au sein de la hiérarchie sociale locale, émiriennes ou expatriées issus de pays du Moyen-Orient ou d'Afrique de l'Est ${ }^{14}$. Lorsque de jeunes émiriens optent pour ce choix de vie, comme nous l'explique un sportif émirien de haut niveau originaire de Ras Al-Khaimah, l'émirat de Dubaï et encore un peu plus l'émirat d'Abu Dhabi s'affirment comme des pôles d'attraction majeurs pour les meilleurs d'entre eux qui espèrent briller. Dans cette configuration, de nombreux jeunes sportifs émiriens des émirats du nord cherchent à les rejoindre pour les moyens financiers dont ils disposent. Né à Ajman, le footballeur international émirien, Khalfan Mubarak, ayant débuté plus jeune dans les rangs du club de Dubaï Al-Ahly est un de ces exemples. Dans ce contexte social, la " génération dorée » du football émirien, qui a permis aux Émirats arabes unis de participer à sa première et seule phase finale de Coupe du monde de football, en 1990, avec une génération qui était issue principalement de familles originaires des villes portuaires des Émirats arabes unis ${ }^{15}$, a laissé la place à une équipe au profil plus régional qui reflète bien les migrations internes à la péninsule Arabique vers le pôle d'attraction que constitue le pays. Cette configuration s'explique surtout par le fait qu'à Dubaï, comme dans le reste des EAU, la pratique sportive est née de l'interaction entre populations étrangères régionales et populations locales. Derrière le discours identitaire des autorités, sur le terrain, la frontière nationale est plus souple et s'adapte aux besoins des fédérations en fonction du niveau de leurs effectifs. 


\section{Une centralité sportive en question: la régionalisation des effectifs nationaux}

Pour bien décrypter cette situation, il faut partir des acceptions juridiques que revêt pour les autorités émiriennes le mot nationalité, il est nécessaire de revenir à la linguistique. Comme le développe Uri Davis dans ses travaux, en arabe, deux mots retranscrivent le concept de nationalité: jinsiyyāt et muwātana, cependant avec des degrés divers d'accès aux prérogatives de la citoyenneté (Davis, 2000). La jinsiyyāt offre l'accès au passeport, au droit de résidence, de travail et de propriété, tandis que la muwātana accorde des droits civils, politiques et socio-économiques (Davis, 2000). Les pouvoirs en place jouent sur ces distinctions sémantiques pour établir des niveaux informels dans l'accès aux droits à la citoyenneté, dans le but d'équilibrer le jeu politique interne à leur société, en fonction des enjeux politiques du moment (Kinninmont, 2013). Ce sont ces différentes échelles de la nationalité qui offrent aux autorités l'opportunité d'ouvrir leurs clubs dès les catégories de jeune, à des athlètes appartenant à des familles originaires de la région auxquels les premiers degrés de la nationalité peuvent facilement être octroyés. Sur les bords du terrain, nous observons alors au sein des effectifs des clubs une mosaïque régionale se mettre en place. Dans nos discussions avec des entraineurs et formateurs, les deux phrases «Oui il est né ici, mais ses parents sont... » ou " Il est arrivé ici jeune et aujourd'hui il est émirien » sont courantes. Cet espace sportif apparaît alors comme un reflet des flux migratoires internes au Moyen-Orient. Le sport devient ainsi pour ces sportifs, souvent étrangers à la communauté émirienne, un vecteur d'ascension sociale, un moyen d'être reconnu par leur performance sur le terrain sportif et, ainsi, de dépasser les frontières juridiques internes à la société émirienne, c'est-à-dire la nationalité par le droit du sang par le père (Le Magoariec, 2019). Par ce biais, ces jeunes sportifs ou sportifs déjà engagés dans une carrière de haut niveau espèrent accéder à nombre de privilèges qu'offre la nationalité émirienne. 
Illustration 3 - Les visages sportifs de Dubaï, d'objet politique local à la vitrine mondiale

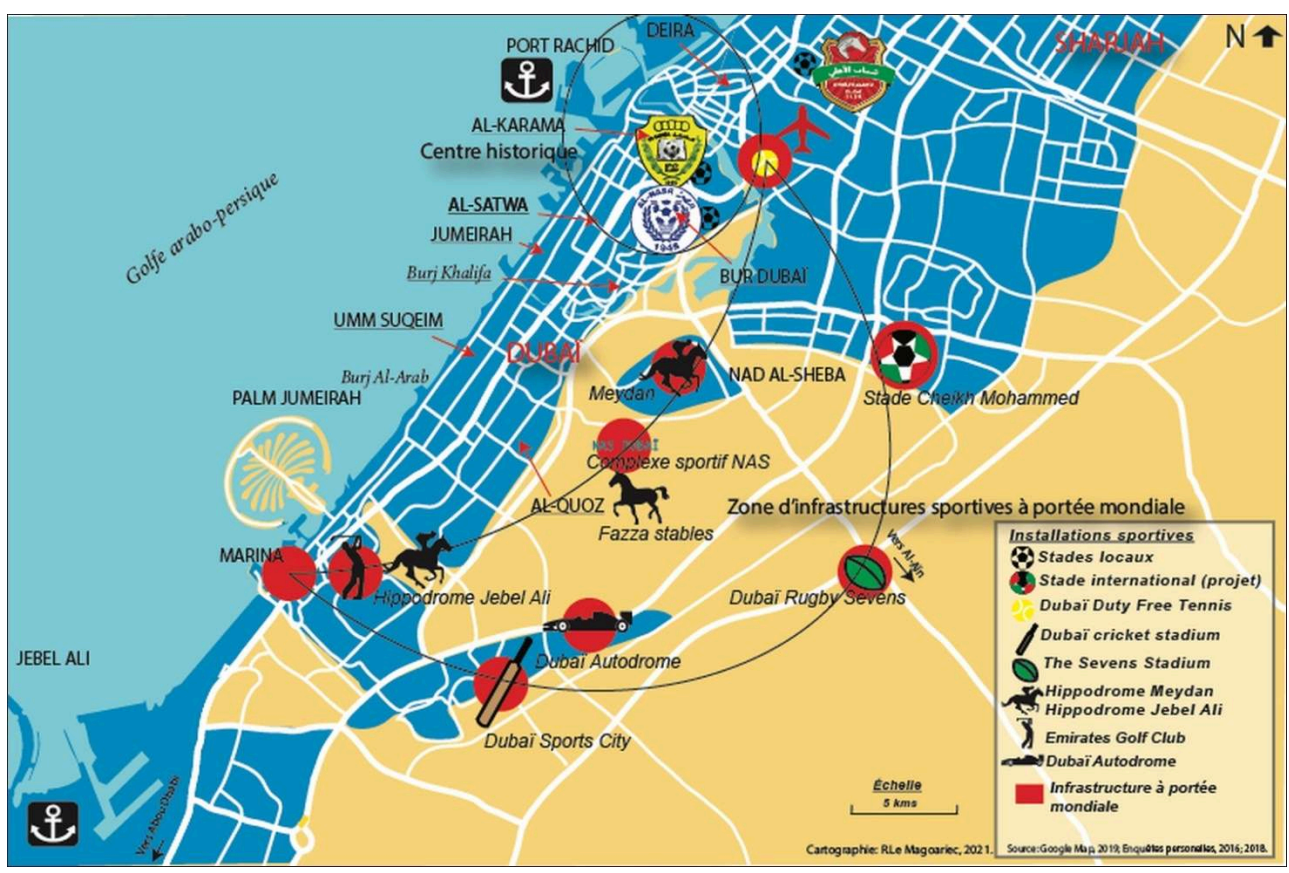

Auteur: R. Le Magoariec, 2021

Parmi les joueurs de la sélection émirienne, nous pouvons citer plusieurs footballeurs issus de familles ayant migré vers Abu Dhabi ou Dubaï. Ali Salem Al-Ameri, Saïd Salem Al-Kathiri, Amer Abdulrahman Al-Hammadi Al-Minhali, Ismail Salem Al-Hammadi AlMinhali ainsi que l'attaquant star de l'équipe nationale, Ali Mabkhout Al-Hajri sont nés aux Émirats arabes unis, à Abu Dhabi ou Dubaï, dans des familles d'origine yéménite et ayant obtenu la nationalité émirienne. De même, né à Abu Dhabi dans les années 1980 de parents omanais originaires de la ville de Sour, l'ancienne star, Ismaïl Matar AlMukhaini Al-Janoubi, a longtemps suscité les espoirs des supporters émiriens. Le cas d'Omar Abdulrahman Al-Amoudi diffère un peu. Né de parents hadramis - de l'Hadramaout, région de l'est du Yémen -, à Riyad, formé dans l'un des clubs phares de la capitale saoudienne, Al-Hilal, il a rejoint le club du sud de l'émirat d'Abu Dhabi, AlAïn, après s'être vu refuser la nationalité saoudienne pour l'ensemble de sa famille, condition que lui assuraient les Émirats arabes unis. Ce sujet, qui touche à l'identité nationale sur un plan politique, reste sensible. Les autorités émiriennes ont, en effet, la volonté de promouvoir « une identité nationale qui fait des origines étrangères d'une part importante des citoyens des EAU un impensé » (Kazerouni, 2017). De ce fait, les révélations par le journal qatarien Al-Charq, en 2013, des origines de plusieurs des joueurs de la sélection émirienne, ont été peu appréciées par le pouvoir émirien. Outre les joueurs issus de familles originaires de différents pays de la péninsule Arabique attirées par le dynamisme des Émirats arabes unis, dans les années 2000, la professionnalisation du football émirien engendre l'ouverture des effectifs de chaque club à quatre joueurs étrangers, ce qui conduit à une timide mondialisation de sa ligue. Face à la stagnation des résultats de la sélection nationale, les autorités décident de naturaliser certains des joueurs professionnels résidant dans le pays depuis plusieurs années et n'ayant, de plus, jamais joué dans la sélection de leur pays d'origine. Cette tentative vise à apporter de la qualité technique et de l'expérience à l'équipe nationale tout en respectant la réglementation de la FIFA. Pour donner un caractère identitaire à 
son entité nationale, des joueurs d'Afrique du Nord ou de l'Est peuvent être ciblés, à l'instar du défenseur de la sélection, né dans la banlieue de Rabat, Ismaïl Ahmed. Dans une logique sportive, la fédération des Émirats arabes unis décide toutefois durant l'année 2020 de naturaliser Sebastian Tagliabue, Caio Canedo et Fabio Virginio de Lima, des joueurs sud-américains brillant dans son championnat depuis plus de cinq ans. Cette décision respecte ainsi le cadre réglementaire de la FIFA adopté en 2008, afin d'éviter des excès notamment de la part de plusieurs pays du Golfe, de proposer la naturalisation à des joueurs sud-américains jouant dans de grands championnats européens.

Illustration 4 - Les Émirats arabes unis, des émirats aux politiques sportives à différentes portées

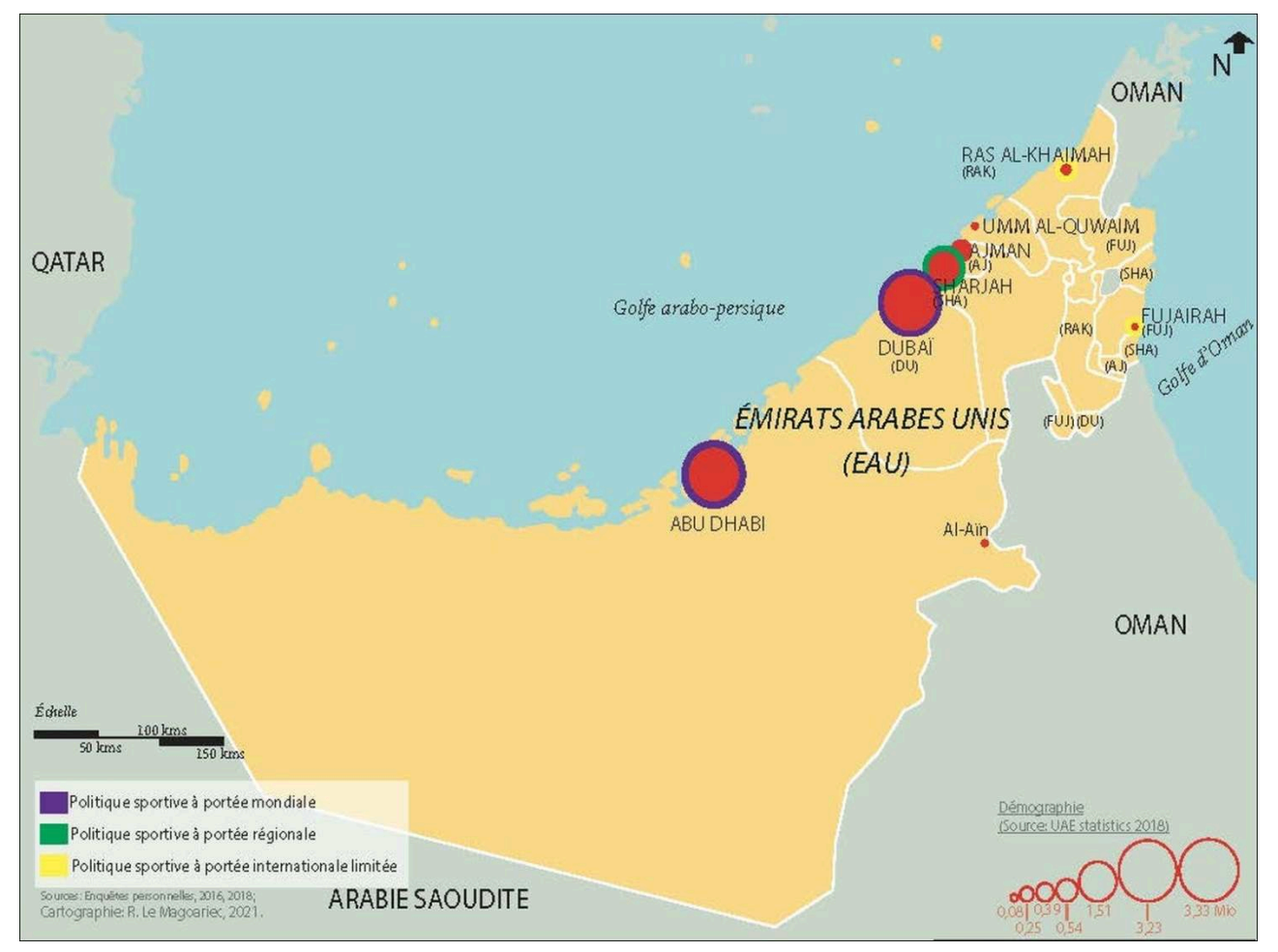

Auteur : R. Le Magoariec, 2021

\section{Un modèle sportif fédéral en recherche de solutions viables}

11 Cette tendance traduit plus globalement un changement qui s'affirme dans le sport émirien. En effet, la délimitation de l'espace sportif fédéral qui se base sur la nationalité tend peu à peu à s'estomper dans les textes même si la réalité de l'accès aux clubs reste sélective. Le Président de la fédération des Émirats arabes unis et émir d'Abu Dhabi, Cheikh Khalifa Bin Zayed Al-Nahyan, a promulgué un décret au début de l'année 2018 ouvrant les clubs nationaux aux enfants nés de parents expatriés sur le territoire émirien et aux enfants de résidents étrangers ${ }^{16}$. Cette ouverture intègre l'aspect de la performance, de nombreux entretiens avec des formateurs font ressortir le fait que de nombreux sportifs émiriens sont maintenus dès leur plus jeune âge, avec ces ligues fermées aux enfants issus de familles d'expatriés ${ }^{17}$, dans une certaine endogamie sportive qui n'incite pas au dépassement de soi et nivelle les potentiels vers le bas. Un caractère qui est contraire à la base intrinsèque de la compétition sportive, qui se 
définit par son ouverture à l'opposition. Cette volonté affichée de bousculer les lignes s'inscrit dans la perspective de mettre peu à peu en concurrence les sportifs émiriens, pour les amener vers le dépassement de soi et ainsi de tenter de les conduire vers l'excellence. Les enfants expatriés apparaissent comme une variable d'ajustement du corps sportif émirien. Plus globalement, cette politique s'intègre dans un projet politique de société. Ce projet passe par exemple par l'instauration, en 2014, d'un système de conscription obligatoire ${ }^{18}$. Au-delà de la volonté d'impulser un retour à la culture de l'effort physique, il faut noter que la conscription s'inscrit dans un contexte politique particulier en ces temps de révolutions et de contre-révolutions. Par la conscription, il s'agit pour les autorités fédérales d'imprégner la jeunesse émirienne de nationalisme émirien autour des grandes figures de l'État, un sentiment récent au sein de la fédération, tout en ayant la volonté de maintenir cette jeunesse dans la discipline face au pouvoir. Enfin, ce décret s'inscrit dans une logique économique régionale qui tend, de plus en plus, vers une libéralisation économique du domaine sportif national. Le système sportif qui se forme autour des clubs gouvernementaux est une émanation de l'État-providence. De ce fait, ce secteur ne repose pas sur l'apport financier des sponsors ou des droits télévisés mais bien sur les finances provenant du budget des gouvernements fédéral ou fédérés ainsi que de personnalités issues des familles régnantes proches ou à la tête de certains de ces clubs. Ce système pèse sur les finances, pour se maintenir, il nécessite à court ou moyen terme d'être réformé ${ }^{19}$. Lors de la présentation de ce projet d'ouverture, les autorités ont en effet insisté sur le fait que les clubs étaient dans leur bon droit en recourant à des frais d'inscription pour les enfants étrangers rejoignant leur entité. Récente, cette décision peut questionner quant à la forme qu'elle prendra réellement au sein du secteur sportif gouvernemental historiquement réservé aux Émiriens. En effet, les expatriés oseront-ils dépasser les lignes de la segmentation sociale qui restent pour le moment bien ancrées? De plus, une fois ce premier pas engagé, les autorités fédérales permettront-elles de décloisonner le football professionnel émirien? De fait, chaque club est soumis à un quota de joueurs étrangers fixé à dix, l'équipe ne peut aligner sur le terrain que quatre de ses joueurs, parfois cinq en même temps, parmi lesquels un sportif issu de la confédération asiatique et trois ne relevant pas de cette zone sportive,. L'objectif étant de préserver des places pour les sportifs nationaux pour favoriser leur éclosion au plus haut niveau. Il est encore difficile de voir clair face à l'opacité et à la centralité de la décision, mais la logique engagée devrait amener à la poursuite de l'ouverture du sport national aux non-golfiens tout en maintenant un équilibre identitaire fragile entre sportifs golfiens et étrangers. Cette tendance de plus en plus visible dans la région est un sujet de questionnement récurrent chez de nombreux entraineurs étrangers.

\section{Entre tape-ball cricket et cricket, l'influence du Commonwealth}

Derrière le système sportif émirien qui reste en grande partie cloisonné, l'activité physique des expatriés est éclairante. Tout en étant proches spatialement, ces pratiques ou entités sportives que fréquentent les expatriés et leurs enfants sont en réalité éloignées de la vie sportive nationale, elles s'inscrivent dans des imaginaires nationaux voire mondialisés différents. Nous évoquerons ici une pratique sportive déterritorialisée qui se décline à différents échelons. Le sport expatrié se définit par 
une opposition entre espaces de jeu informels et formels. Les expatriés aux hauts revenus jouent sur des terrains sportifs en gazon, accomplissent leur effort dans les salles de sport et de fitness prévues à cet effet, tandis que le flot d'expatriés aux bas revenus souvent originaires des pays sud-asiatiques s'adonnent au sport lorsqu'ils en ont le temps et l'énergie suffisante, sur des parkings ou des friches industrielles. Dans l'observation de ces dynamiques, à travers les usages de l'espace sportif c'est une logique de centre-périphérie qui apparait.

Illustration 5 - Promotion d'un événement international de cricket (Dubaï, février 2016)

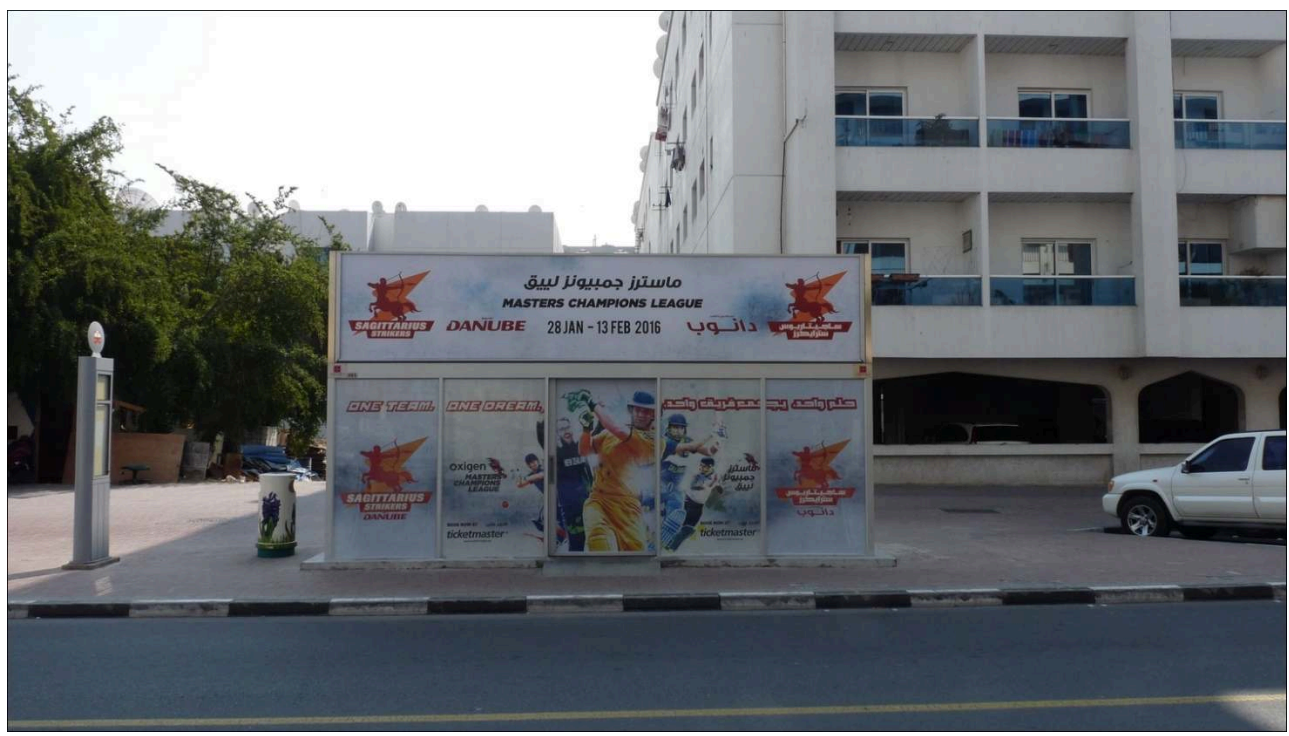

Arrêt de bus climatisé situé dans le quartier de Deira recouvert d'une affiche faisant la promotion d'un événement international de cricket réunissant plusieurs stars mondiales de ce sport.

Auteur: R. Le Magoariec.

\section{Un espace marqué par l'omniprésence de la culture du cricket}

Reflet des importantes relations nouées par le passé entre le sous-continent indien et les rives du Golfe, dont Dubaï, le cricket apparaît aujourd'hui comme l'émanation même de ce phénomène de fragmentation socio-spatiale de l'espace sportif dubaïote. À Dubaï, deux types de pratiques éclosent: le cricket et le tape ball cricket. La passion pour le cricket se développe à différents niveaux et émerge sur des terrains qui se différencient de par leur aménagement.

14 Le cricket s'est réellement imposé au lendemain de la Seconde Guerre mondiale sur le territoire de l'émirat à travers les communautés issues du Commonwealth. Auparavant, des terrains avaient déjà été construits à proximité des différentes bases des forces armées britanniques présentes sur le territoire des États de la Trêve ${ }^{20}$, pour qu'elles puissent s'exercer sur leur temps libre au cricket (Morgane, 2007). Toutefois, c'est surtout l'importante présence de populations originaires du sous-continent indien, souvent issues de milieux marchands, en particulier autour des ports de Dubaï et de Sharjah, qui favorise l'émergence de ce jeu. Le tissu social qui compose le territoire de Dubaï est ainsi propice à son développement. L'accroissement de Dubaï, tout au long des décennies 1960 et 1970, dans son rôle de hub régional, accentue son attractivité pour des populations en provenance d'Asie du Sud. Il faut noter que ces logiques 
migratoires s'inscrivent, de plus, dans un contexte de Guerre froide, et de surcroit, de "Guerre froide arabe », la main d'œuvre issue de pays du Proche et Moyen-Orient se trouve de ce fait de moins en moins privilégiée par les principautés du Golfe, dont l'émirat de Dubaï, qui se tournent alors davantage vers une main d'œuvre asiatique moins onéreuse (Davidson, 2007 ; Chalcraft, 2012 ; AlShehabi, 2015).

Illustration 6 - Match de cricket de la Pakistan Super League (Dubaï, 2018)

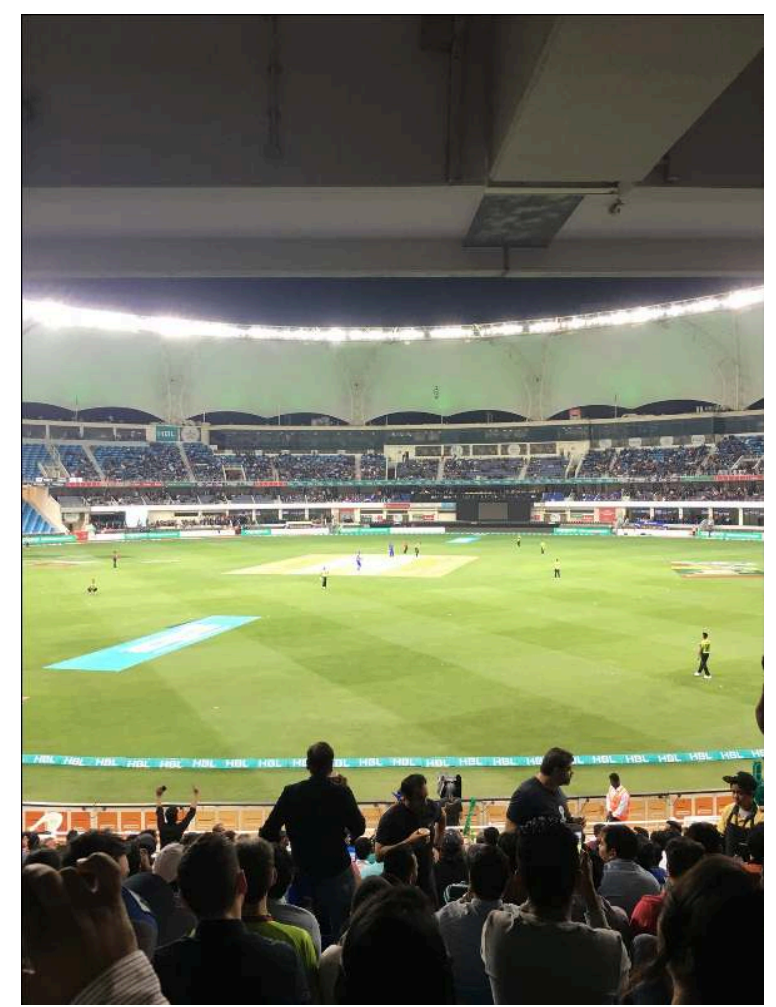

Match opposant au sein de l'International Cricket stadium de la Dubaï Sports City, l'équipe des Lahore Qalandars aux Karachi Kings.

Auteur : R. Le Magoariec.

En outre, les relations diplomatiques construites par plusieurs émirats avec des pays du sous-continent indien, auxquelles s'ajoutent les relations développées à l'échelle même de familles émiriennes avec cette zone géographique (Vora, 2013), participent à ancrer le cricket au sein de plusieurs émirats des Émirats arabes unis, au premier rang desquels Sharjah et Dubaï. Dans ce cadre, au cours des années 1980, deux stades sont conçus dans l'optique d'accueillir des matchs internationaux, à Sharjah, puis à Dubaï. Derrière ces projets se trouve un passionné de culture indienne, Abdulrahman Boukhatir. Amoureux de cricket, cet homme d'affaires émirien a fait ses études au Pakistan et il apparaît comme étant un acteur central dans la promotion de ce sport au sein des instances du pays. Le cricket, comme pratique formelle, qui s'est peu à peu solidifié dans les années 1960-1970 autour de clubs, se structure désormais autour d'une fédération fédérale qui rejoint, dans cet élan de construction, l'International Cricket Council, en 1990. Toutefois, excepté quelques Émiriens qui pratiquent ce sport, le cricket reste l'apanage des résidents issus du Commonwealth. Les communautés du souscontinent indien demeurent le moteur de ce sport, à Dubaï, et plus globalement dans l'ensemble des Émirats arabes unis ${ }^{21}$. Au sein de la fédération, les Émiriens apparaissent comme décideurs sur le papier, mais derrière les noms présents dans l'organigramme, 
excepté pour quelques Émiriens intéressés par ce sport, ce sont bien plus les membres issus des communautés du sous-continent indien qui gèrent ce domaine ${ }^{22}$. Du fait de l'absence d'Émiriens, ce sport n'est pas financé par les structures étatiques ${ }^{23}$.

Illustration 7 - Public assis dans les tribunes de l'International Cricket stadium de la Dubaï Sports City lors d'une rencontre de la Pakistan Super League (Dubaï, 2018)

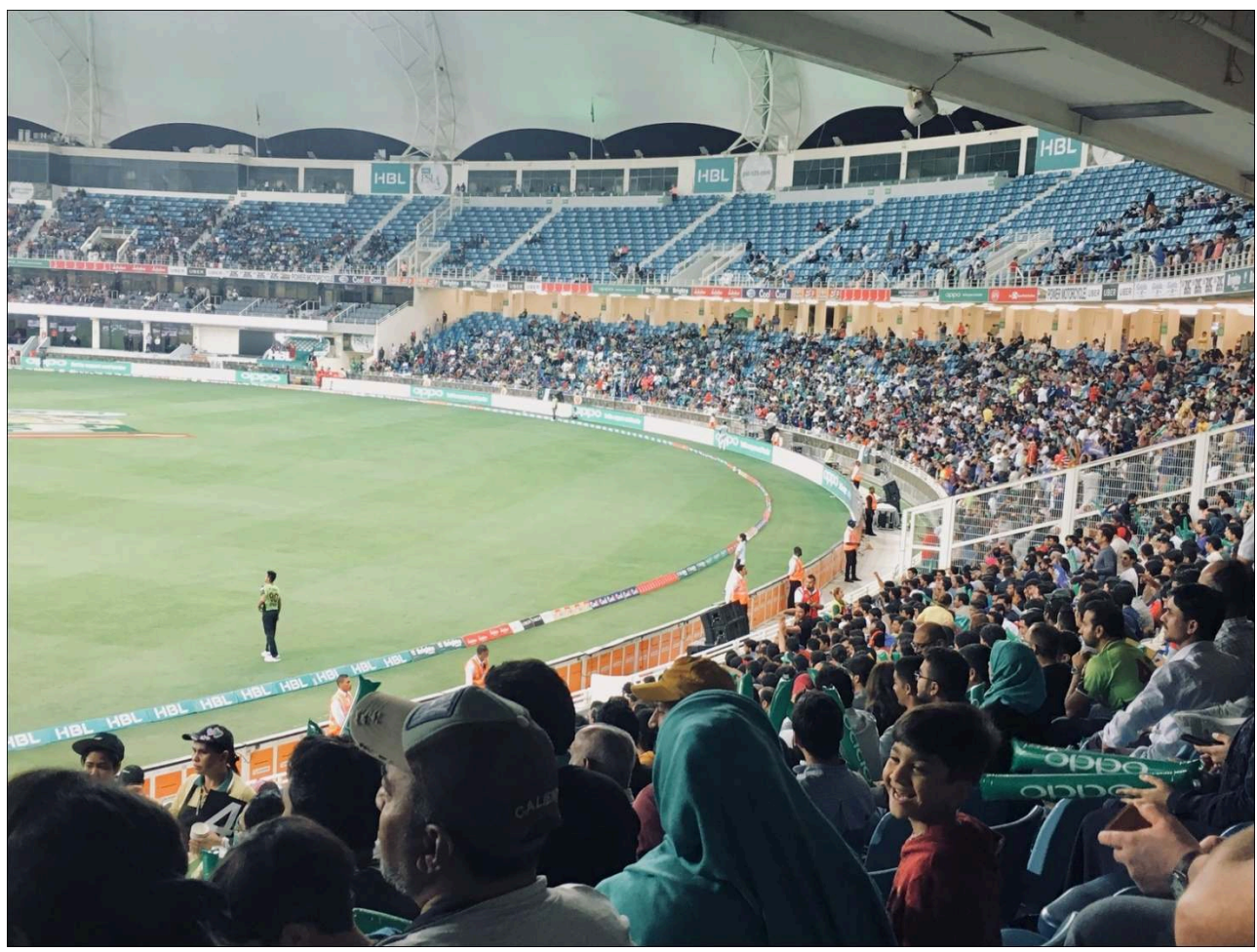

La passion pour le cricket au sein du pays témoigne de la place qu'incarne les Émirats arabes unis, au premier rang desquels l'émirat de Dubaï, pour les sociétés du sous-continent indien. Dubaï apparaît, aujourd'hui, comme un pôle mondial majeur du cricket.

Auteur: R. Le Magoariec.

\section{Le terrain gazonné, un accès déterminé par la classe sociale}

Accéder aux terrains de cricket demeure un privilège de classe. Les frais d'inscription se dressent comme une frontière sociale à l'accès au terrain officiel. Dès lors, ce sont les expatriés aux hauts revenus qui intègrent cette architecture. Certains d'entre eux issus de la classe moyenne optent pour pratiquer ce sport dans des clubs de l'émirat voisin de Sharjah, le coût d'inscription y étant souvent moins élevé ${ }^{24}$. Des frais qui varient en fonction des catégories d'âge, tandis qu'à Dubaï les prix se situent entre 1000 AED et 3 $000 \mathrm{AED}^{25}$ pour une année, à Sharjah le coût d'une inscription est de l'ordre de $650 \mathrm{AED}^{26}$. Ce tissu sportif se structure également, à partir des années 2000, autour d'entreprises qui participent par la création d'une équipe à une professionnalisation de sa pratique ${ }^{27}$. Elles contribuent à l'échelle du pays au développement de ligues. Cette assise se constitue aussi autour d'hommes d'affaires. Abdulrahman Boukhatir et le riche entrepreneur indien, Shaji Ul Mulk, domicilié à Dubaï, tous deux membres de l'Emirates cricket board, se distinguent ainsi par leur engagement dans la fondation de compétitions qui se déroulent, à Sharjah, Dubaï ou Abu Dhabi, sous différents formats. Ils poursuivent l'objectif de maintenir une dynamique croissante autour de ce sport au 
sein du pays. À l'instar de Shaji Ul Mulk et de différents responsables rencontrés dans les coulisses du cricket à Dubaï, d'origines indienne ou pakistanaise, aux Émirats arabes unis les classes moyennes et aisées issues du sous-continent indien sont motrices dans la marche du cricket. Nés à Dubaï ou Sharjah, ayant migré dans des logiques économiques et/ou sportives, les joueurs présents sur les terrains de cricket témoignent de la place qu'incarne le Golfe arabo-persique pour le sous-continent indien. Dubaï apparaît comme un prolongement de cette région (Vora, 2013). La pratique de ce sport et les événements qui se déroulent autour du cricket au sein de cette conurbation englobant les villes de Dubaï, Sharjah et Ajman, s'inscrivent dans ce contexte. Pour les sportifs du sous-continent indien, le pays se transforme en un possible débouché pour la poursuite de leur carrière. Iqbal Husseïn Chaudhry, l'actuel capitaine de la sélection qatarienne d'origine pakistanaise, a fait ses études à Dubaï et connait bien les réalités internes au cricket émirien. Lors de notre rencontre à Doha, il nous explique que les conditions d'entrainement aux EAU, même si les joueurs sont considérés comme semi-professionnels, sont proches de celles de professionnels. Les joueurs ont des horaires aménagés, "nous pouvons dire qu'ils sont payés pour s'entrainer, ils sont placés dans les meilleures conditions pour performer $»^{28}$. Dans le sens inverse, pour les grandes équipes de cricket du sous-continent indien, ce pays devient un vivier de potentiels talents à surveiller de près. Ainsi, des académies de certains grands clubs s'implantent à Dubaï pour suivre les jeunes joueurs de ces communautés issues d'Asie du Sud.

\section{Les périphéries, champs du tape ball cricket}

17 Dans cette configuration, la masse d'employés à bas revenus est quant à elle reléguée. Face à cette frontière, ces passionnés de cricket s'emparent des espaces de la ville sans fonctionnalités précises, les friches industrielles et parkings, qu'ils adaptent en terrains de jeu dédiés à la pratique du tape ball cricket, le cricket des rues. Ils sont magasiniers, conducteurs de Uber ou plus rarement de taxi, travaillent à l'aéroport, dans des bureaux ou dans les cuisines de petits restaurants communautaires, tous ces hommes venus à Dubaï pour rapporter un mince pécule à leur famille restée au pays, investissent, les vendredi matins ou après-midi, les parkings ou friches industrielles de la ville, le temps de quelques heures, pour jouer au tape ball cricket $^{29}$. Au sein des quartiers Al-Quoz, d'Al-Satwa, de Deira ou d'Al-Karama, à travers l'imagination de ces personnes reléguées, ces espaces se voient appropriés dans une utilité sociale nouvelle. Dans les échanges avec certains de ces joueurs, c'est la passion du cricket et la reproduction des gestes qu'ils regardent à la télévision depuis leur plus jeune âge qui ressortent avant tout, mais également la perception du terrain informel comme un lieu de sociabilité avec d'autres personnes qui partagent des quotidiens similaires, éloignées de leur cercle familial. Dans ces lieux, la communauté d'origine laisse place à une communauté sociale tout aussi fédératrice. Ils jouent ainsi souvent à proximité de leurs habitats, mais certains d'entre eux viennent parfois d'autres émirats pour échanger des balles avec des connaissances. Il faut noter, toutefois, que certains jeunes joueurs investissent ces espaces dans une volonté d'ascension sociale, dans l'espoir d'être repéré par la fédération de cricket émirienne et, par ce biais, de contourner leurs déterminismes sociaux. Mohammed Naveed, l'ancien joueur pakistanais de la sélection des Émirats arabes unis, a eu ainsi la chance de connaître ce destin en étant repéré lors de tests passés à Dubaï et Sharjah. Bien que poreuse, la frontière entre les terrains 
informels et formels reste marquée, ce sont alors les individus les plus motivés, talentueux et résistants, qui parviennent lorsqu'une mince occasion se présente à atteindre l'espace reconnu par les institutions, le stade de gazon.

\section{Dans les centres, la pratique des expatriés aux hauts revenus: entre bien-être et dépassement de soi}

\section{Dubaï et les expatriés aux hauts revenus : l'accroissement de la mondialisation sportive}

À Dubaï, le tissu économique se développe autour du secteur tertiaire. Dans ce domaine, les fonctions supérieures à forte valeur ajoutée sont l'apanage d'une upper class qui se forme principalement autour des expatriés européens, américains et asiatiques. Ces populations qualifiées amènent avec elles des représentations et usages du sport occidentalo-centrés. La tendance actuelle est clairement au fitness et au healthy life style, découlant des traits de l'american way of life mondialisé. À Dubaï, et plus globalement aux Émirats arabes unis, les expatriés à hauts revenus s'inscrivent dans une société animée par la réussite individuelle. Ce contexte socio-économique contribue à accentuer la place que prend le corps dans leur vie (Vigarello, 2015), ils cultivent ainsi l'image d'un corps performant, synonyme de maitrise de soi, assimilée selon les normes de la société au succès (Queval, 2012). Derrière le sport, c'est ainsi une économie locale de l'effort, du plaisir et du bien-être, qui se développe alors massivement à partir des années 1990 au cœur du projet de Dubaï. Dans le mouvement d'extension urbaine que vit Dubaï, de nombreuses infrastructures sportives sont construites. Les personnes qui ont de hauts revenus peuvent dès lors se payer le luxe d'accéder à des espaces sportifs modernes et fonctionnels. Ce sport émerge à Dubaï à travers les écoles qui accueillent des enfants d'expatriés, les différents clubs ou académies et les salles de fitness qui se multiplient dans la ville. Ces lieux ont parfois pour déterminisme une origine spatiale commune ou sont simplement ouverts à toute personne ayant les moyens de payer l'adhésion. Ces frais diffèrent en fonction des pratiques sportives, ils sont en moyenne de l'ordre de 1000 AED à 2500 AED. Ces académies ou clubs sportifs, qui se définissent par rapport à une origine, le font souvent dans l'optique de mettre en avant la langue et les méthodes d'apprentissage qu'ils emploient au sein de leur structure, mais c'est aussi une façon de tisser du lien social entre des personnes de même culture. Américains et Européens, familles aisées issues de pays émergents, ces protagonistes vivant à Dubaï et éloignés de leur société d'origine sont à la recherche des infrastructures adéquates à la bonne pratique de leur sport de prédilection. Certains sports ont été intégrés à la ville par des expatriés amateurs, passionnés par une discipline précise ${ }^{30}$; ce sont d'autres fois des professionnels qui ont vu en Dubaï un espace propice au développement de leur activité économique ${ }^{31}$; enfin, des membres appartenant aux familles régnantes ont pu apprécier un sport particulier et avoir eu la volonté de le développer sur leur territoire, à l'image de l'essor soudain du jiu-jitsu brésilien et d'autres sports de combat tant chez les Émiriens que chez les expatriés. Dubaï a ainsi été une voie d'entrée de nombreux sports sur le territoire émirien et a eu une influence importante sur l'ensemble des Émirats arabes unis. Cependant, c'est à partir de l'émirat voisin d'Abu Dhabi que se sont diffusées à Dubaï certaines pratiques sportives, comme en témoigne la croissance du jiu-jitsu brésilien et du hockey sur glace. De par leur démographie et la présence 
d'expatriés aux hauts revenus, ces deux pôles majeurs des EAU jouent un rôle fondamental dans la diffusion de nouvelles pratiques sportives centrées sur la nature au sein de l'arrière-pays de plusieurs émirats du nord. Les massifs montagneux du Hajar sur les territoires de Ras Al-Khaimah, de Fujairah et de Sharjah sont aménagés pour permettre de s'adonner à ces différentes activités physiques tels le trail, le VTT, la randonnée et l'escalade.

Cette pratique sportive apparaît pour ces adultes et enfants étrangers comme un moment de loisir, de bien-être, de performance et de sociabilité loin de leur pays d'origine ; la sociabilité qui en découle est d'autant plus capitale au cœur de cette ville rythmée par son injonction à la réussite économique et sociale qui s'impose aux employés étrangers. La pratique sportive permet ainsi de combler leur temps libre de moments de loisir, de maintenir leur motivation par l'effort accompli en compagnie de coéquipiers qui connaissent des réalités similaires, pour mieux se replonger dans le travail. Par ailleurs, certaines femmes sans emploi, ayant suivi leur mari, voient dans les salles de fitness et le sport l'opportunité de se tisser des liens de sociabilité ${ }^{32}$. Cloisonnées hors du secteur du travail, il s'avère souvent plus difficile pour elles de se créer de telles opportunités. En plus de s'affirmer comme un lieu de maintien en bonne santé ou de dépassement de soi, le terrain sportif devient pour ces femmes une manière d'exister dans cette nouvelle vie à l'étranger. Plus globalement, le terrain sportif peut apparaitre comme un lieu pour avoir la sensation de faire communauté (Queval, 2004). Dans une série d'entretiens portant sur la pratique du sport, menés auprès de trente expatriés aux hauts revenus vivant à Dubaï, ou ayant vécu dans l'émirat, le champ lexical de la "sociabilité " ressort très distinctement dans chacun de ces échanges lorsqu'il s'agit d'aborder la raison de leur fréquentation de l'espace sportif. Au-delà de la condition de ces femmes expatriées, dans cette société régie par un système capitaliste débridé, par l'imaginaire d'un règne du sans limite, la question du paraître est primordiale dans le milieu des expatriés aux hauts revenus, il s'agit de cultiver une image positive vis-à-vis de leurs collègues, clients ou concurrents. "L'apparence est une construction où l'individu se fait et se parfait lui-même» (Vigarello, 2012); au cœur de l'économie mondiale contemporaine, le culte du corps revêt une place particulière. Dans ce système, l'individu est poussé à se montrer aux yeux de ses pairs dans un état de santé performatif (Chicchi, Simone, 2017). Les salles de fitness ou les pratiques à la mode qui en découlent comme le cross-fit, s'inscrivent dans cette configuration sociale, elles participent dès lors pleinement au développement économique de la ville. Au travers de ce milieu social, c'est l'économie du bonheur qui émerge. Il s'agit d'une offre conçue comme un palliatif et une échappatoire pour une catégorie sociale qui mène, à Dubaï, une vie active intense et fatigante. Dubaï est ainsi devenu un pôle majeur pour les coachs sportifs du monde entier en quête de nouveaux challenges et de profits financiers importants. Dans la définition de son attractivité, ces activités sportives à la mode apparaissent comme des moteurs dans la communication de Dubaï pour plaire aux travailleurs expatriés en recherche d'une vie dynamique agrémentée d'activités assimilées à des pratiques menant au bien-être. Dans l'affirmation de cette politique, le pouvoir a même fait du fitness un de ses leitmotivs en programmant chaque année une semaine dédiée au fitness. L'objectif est d'insuffler du bien-être, du bonheur, dans sa société tant dans les milieux expatriés que nationaux. Dubaï a fait de la culture mondiale du healthy un fil conducteur de la vie dans sa cité. Plus largement, il l'a aussi conçue comme un vecteur de son image mondialisée. 
20 Cet ancrage spatial du «bien-être » émerge à Dubaï suivant une logique polycentrique, puisque différents acteurs à la fois gouvernementaux et privés participent à l'instauration de cette dynamique. Lors d'un entretien mené avec une femme d'affaires travaillant dans le secteur de la voile à Dubaï, celle-ci insista d'ailleurs sur l'importance que revêt le secteur privé dans le développement du sport au sein de l'émirat de Dubaï. Elle nota que cette politique locale diffère de celle de son émirat riverain Abu Dhabi, qui a contrario s'affirme plus entreprenant dans l'édification de son tissu sportif. À Dubaï, le Dubaï Sports Council est central, il contrôle la création de nouvelles infrastructures dédiées à ce secteur et participe lui-même à la construction d'espaces multisports, par exemple celle du complexe Dubaï Sports World. Derrière cette autorité étatique, ce sont surtout les acteurs privés qui se démarquent. Dans cette catégorie, nous pouvons distinguer trois types d'acteurs privés.

\section{Les piliers de l'industrie du fitness}

D'une part, les hommes d'affaires issus de grandes familles marchandes de l'émirat. Ils conçoivent ce domaine comme une extension de leur marché existant à un nouveau secteur économique attractif. Parmi eux, nous pouvons citer dans cette catégorie des membres des familles Al-Ghurair, Al-Tayer et Al-Futtaim. Ainsi, le groupe Al-Futtaim a fait de ce domaine l'un des piliers de son projet immobilier bordant la crique, le Dubaï Festival City.

$22 \mathrm{Au}$ sein de ce paysage des clubs de fitness émerge, d'autre part, un protagoniste de l'économie golfienne, à savoir Micky Jagtiany. Ce riche homme d'affaires né à Koweït City dans une famille marchande indienne, vit aujourd'hui, à Dubaï, il est à la tête d'un puissant conglomérat qu'il a fondé en 1973, au Bahreïn : le groupe Landmark. Percevant la rapide évolution des sociétés de la péninsule Arabique et les potentielles retombées du changement de modèle de société, il étend ses activités au marché du « sport bienêtre ». Il lance, à Dubaï, en 1993, sa chaîne de club de fitness, Fitness first.

Enfin, dernière catégorie, des entrepreneurs occidentaux qui perçoivent dans la sociologie de Dubaï une clientèle potentielle en recherche d'espaces de détente et de nouveautés. Dans ce cadre, ces acteurs ont plusieurs possibilités : soit ils choisissent de s'intégrer aux logiques juridiques émiriennes, en ayant l'obligation de trouver un sponsor et partenaire émirien qui possédera alors $51 \%$ des parts de l'entreprise créée, soit de s'inscrire dans des zones franches qui l'extraient du droit de l'émirat. Outre son importante démographie étrangère et son modèle de société, le contexte climatique de l'émirat contribue lui aussi à l'essor de ce marché des salles de fitness. D'avril à octobre, en effet, faire du sport en extérieur devient compliqué, le sportif est confronté à des conditions météorologiques rudes caractérisées par des températures élevées avoisinant les 40 à 50 degrés Celsius, un fort taux d'ensoleillement et des taux d'humidité variant en moyenne entre 60 à 70 pour cent. Ce niveau d'humidité freine le mécanisme de sudation $\mathrm{du}$ corps humain, ce qui constitue un obstacle à son refroidissement et rend inhumaine la pratique d'une activité physique en extérieur. Ainsi, ce facteur est également à prendre en compte pour comprendre l'engouement pour ces lieux sportifs en intérieur. Basé sur les loisirs, cet environnement consumériste, apparaissant comme un possible nœud de sociabilité, contribue à nourrir l'attractivité de l'émirat. 


\section{Un domaine sportif en pleine expansion : un pôle de richesses, entre offre et demande} sportive répond à la demande. Le football, sport mondialement pratiqué, reflète pleinement cette réalité d'un marché "anarchique», commente un professionnel ${ }^{33}$ travaillant dans le football de haut niveau au sein des sociétés du Golfe. Le football laisse apparaître un ensemble d'institutions bien éloignées de la réalité des clubs gouvernementaux réservés aux citoyens émiriens. Cet ensemble se compose, d'une part, d'académies qui proposent généralement des programmes sérieux pour des enfants d'expatriés avec des professionnels diplômés compétents qui ne s'appuient pas forcément sur un marketing particulier, bien qu'elles puissent être dirigées par d'anciens footballeurs professionnels européens. Tandis que d'autres académies mettent surtout en avant des noms de clubs prestigieux ou d'anciens joueurs majeurs, à l'image de l'académie du FC Barcelone, du Real Madrid FC, de la Juventus Turin, du PSG, de la Liga, ou encore, de l'académie rattachée au projet immobilier de la Dubaï Sports City qui a fait appel à l'ancien footballeur du Real Madrid, Michel Salgado, pour diriger son entité. L'objectif est alors de s'appuyer sur ces noms réputés pour attirer une population avec un pouvoir d'achat important. Ces institutions font souvent miroiter aux jeunes aspirants et à leurs parents le rêve d'une carrière professionnelle à travers un logo ou un nom légendaire assimilé au succès. Une telle issue n'est pas impossible mais l'objectif premier de la présence de tels groupes reste surtout le gain d'argent. Ces entités ne sont pas présentes à Dubaï dans un objectif de détection, cependant si l'occasion se présente, ils pourront proposer aux jeunes joueurs de rejoindre le centre de formation de l'institution mère, ce qui reste toutefois peu probable ${ }^{34}$. Quoiqu'il en soit, si ces joueurs, qui garnissent les dizaines d'académies de la ville, ont la chance d'atteindre l'élite de leur sport, ils en feront l'expérience loin de Dubaï. Excepté dans le cadre de sports individuels, les jeunes expatriés qui espèrent atteindre le haut niveau seront dans l'obligation de rejoindre des clubs européens, nord ou sud-américains, en fonction de leur origine et ambitions futures. Les clubs professionnels locaux, contraints par les quotas de joueurs étrangers présents au sein de leurs effectifs, préfèrent en effet pour leur part parier sur des joueurs étrangers plus matures, déjà formés, frileux à l'idée de prendre le risque d'investir sur un joueur qui n'a pas encore l'expérience du haut niveau ${ }^{35}$.

Comme nous le confie un agent de joueurs français implanté à Dubaï depuis plusieurs années, cette métropole, en tant que hub mondial, est propice pour négocier des affaires. Elle possède des connexions aériennes mondiales, il est donc facile de s'y rendre et de repartir. L'hiver, le climat est agréable et tranche avec la météo pluvieuse de nombreux pays du nord. Enfin, il insiste bien sur le fait que l'offre sportive conçue par les entreprises de construction semi-étatiques ${ }^{36}$, proposant un panel de sports traditionnellement réservés à la bourgeoisie : golf, tennis et polo, est pensée pour fabriquer un cadre favorable aux milieux du business. Ce constat pourrait à lui seul faire l'objet d'une étude approfondie tant les aspects soulevés sont vastes sur cette question. 


\section{Un pôle sujet aux influences mondiales, Dubaï guidé par la politique du « bien-être »}

Au-delà de cette fragmentation socio-spatiale, les lignes de distinction qui émergent au sein de la ville de Dubaï ne sont pas figées et parfois perméables, dans certains cas le terrain sportif apparaît comme une passerelle inter-culturelle. Dans cette ville mondiale, plusieurs phénomènes sociaux ont lieu autour de ce domaine. D'une part, la société émirienne entre en interactions avec certaines pratiques importées dans le pays par les expatriés occidentaux ainsi que le développement de pratiques de la jeunesse émirienne qui s'ouvre au monde en voyageant de plus en plus. Ce sont, d'autre part, certains hommes de pouvoir à l'image du prince héritier de Dubaï qui participent à cette transmission en incitant la jeunesse à s'exercer à la pratique de certains sports, comme le cyclisme et le fitness. Cette posture s'explique, d'abord, par le fait que la société émirienne de Dubaï est frappée par des taux de surpoids importants se situant aux alentours de $70 \%{ }^{37}$. Les institutions fédérales et fédérées prennent en compte cette composante pour améliorer le problème de la santé publique qui reste un frein à l'épanouissement de nombreux Émiriens. Lors de nos différents entretiens avec des acteurs rattachés aux ministères du sport de Dubaï et d'Abu Dhabi, nous remarquons l'assimilation fréquente du sport aux mots "bien-être" et "bonheur", cet emploi ressort souvent des discours des responsables émiriens, la recherche de l'épanouissement de leurs citoyens est évidente. À travers cette notion marketing, il s'agit également de projeter une image mondiale séduisante autour des tissus urbains de Dubaï et d'Abu Dhabi. Influencés par de grands noms de la psychologie positive, les Émirats arabes unis créent, en 2016, un ministère du Bonheur. Grand artisan de cet axe politique, l'émir de Dubaï lance ensuite, en 2017, le World Happiness Council, un think tank qui a pour but de nourrir la réflexion autour des notions de «bonheur » et de "bien-être ». Cependant, dans un second temps, cette orientation questionne. En effet, derrière ces mots flous des autorités, cette politique peut être analysée à travers un regard décalé et comprise à la lecture des travaux d'Eva Illouz et d'Edgar Cabanas comme un moyen de poursuivre une finalité bien moins visible, celle de l'obéissance par le bonheur (Illouz, Cabanas, 2018). Cette politique émerge dans un contexte régional tendu, la quête de la stabilité future du pays apparaissant comme sa finalité première. Séduisante la notion de "bienêtre" se transforme ainsi en un soubassement visant à créer un cadre qui favoriserait un tissu social heureux dans le but d'éviter toutes contestations à venir. Les figures du pouvoir s'engagent pleinement en ce sens. À l'instar de Martin Seligman, considéré comme l'un des pères fondateurs de la psychologie positive, ils s'entourent de grands noms de ce courant pour être conseillés. 
Illustration 8 - Piste de course à pied aménagée en bordure de plage dans le quartier de Jumeirah (Dubaï, 2018)

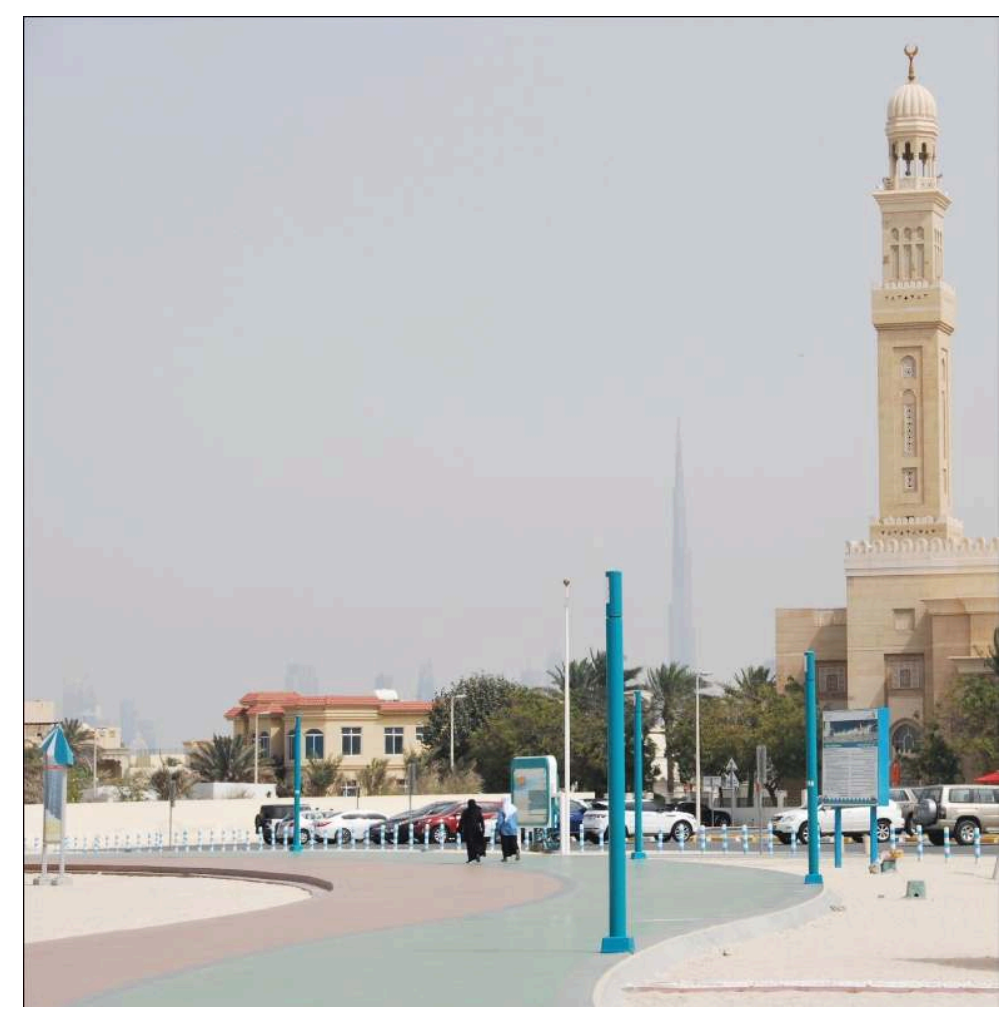

Au loin, deux femmes marchent, la marche à pied reste la discipline favorite pour de nombreux Golfiens ce qui traduit la perte de vitesse de l'activité physique, au cours des quarante dernières années, au sein de la société émirienne face à des changements multifactoriels : étalement urbain, modèle de société tout moteur, alimentation, politique de redistribution économique.

Auteur : R. Le Magoariec.

\section{Conclusion}

Objet stratégique, à Dubaï, les terrains sportifs s'affirment comme des instruments politiques. La traversée de cette métropole mondialisée, quadrillée par les réseaux routiers, donne à voir un paysage sportif morcelé. Les lieux et pratiques qui prennent forme en son sein se caractérisent par leur diversité, c'est la « cité monde » qui apparaît au travers des espaces sportifs de la ville. Cette promenade au cœur de ces lieux permet d'observer les différentes temporalités qui ont façonné la vie sportive, de lire les interrelations entre des acteurs présents sur le terrain et les règles qui encadrent son accès. Différentes couches s'agrègent les unes aux autres, elles sont le produit des phases de migrations qui se sont succédées au cours du XXe siècle, autour de ce pôle régional en pleine croissance. Sur ce territoire, ce sont plusieurs systèmes et pratiques qui se superposent, qu'ils soient formels ou informels. Au cœur des anciens quartiers siègent toujours les clubs historiques de l'émirat. Conçus comme des vecteurs de l'Étatprovidence dans les années 1970, ils donnent de la visibilité au pouvoir au sein de l'espace et s'affirment comme l'un de ses prolongements. Dans l'extension urbaine de la ville de Dubaï, c'est le visage de son attractivité qui se dessine. Les expressions sportives qui se déclinent alors sont multiples et émergent au sein d'un espace sportif fracturé. Cette situation est le produit de l'adaptation de son économie aux contours du 
système financier mondial pour subsister. Les politiques engagées par l'émir Rachid Bin Mohammed Al-Maktoum, dans les années 1970, puis par ses fils, dont Mohammed Bin Rachid Al-Maktoum, prince héritier et actuel émir, principal artisan du Dubaï d'aujourd'hui, ont conduit à faire de la ville un pôle mondialisé central. Cette centralité ressort au sein de ces différentes strates sportives. Dans une économie mondiale guidée, depuis les années 1980, par la doctrine néolibérale, les dynamiques d'exclusion se sont intensifiées. Le panorama qu'offre ce hub mondial, laisse à voir ces logiques de relégation, la pratique sportive en est une illustration.

Le sport s'est ainsi structuré selon des lignes de fractures socio-spatiales fondées sur le capital économique et sur une grille de lecture communautaire ainsi que sur une délimitation identitaire qui prend sa source autour de la politique d'État-providence. Les strates sportives qui se forment à Dubaï peuvent ainsi être interprétées à travers deux niveaux, l'un, horizontal, qui s'inscrit dans des logiques sportives intrinsèques au système étatique émirien et l'autre, vertical, qui se forme autour d'une diversité sportive façonnée par les populations expatriées, cette typologie est le fruit de l'attraction mondiale de la cité-émirat. La frontière reste ténue entre ces deux échelons, le deuxième exerçant une influence unilatérale sur le premier. En effet, le tissu social mondialisé de Dubaï et plus largement des EAU ouvre la société locale à de nouvelles cultures sportives. Derrière l'imaginaire sportif qui émane du nom Dubaï, du fait de l'intensive communication mondiale développée par l'émirat, se cache une autre réalité sportive. Plusieurs facteurs de fragilité ressortent des terrains de recherche étudiés. Les rapides et nombreuses mutations des modes de vie vécues par la population émirienne, depuis les années 1970, représentent notamment des freins systémiques au développement du sport qui était alors perçu par les pouvoirs en place comme un vecteur de modernité et d'unification. D'autres éléments endémiques viennent corroborer ce constat de fragilité, en particulier à Dubaï, très liée aux fluctuations du système financier mondial dont la métropole tire l'essentiel de ses richesses. Face à une captation de populations extérieures nombreuses mais peu ancrées dans le territoire et un système interne mué par des dynamiques contradictoires, l'éventualité d'un ébranlement économique est un élément central dans les problématiques des dirigeants actuels. Le système est sans cesse en mutation, dans une instabilité permanente, qui jusqu'à présent n'a pas su trouver son équilibre.

\section{BIBLIOGRAPHIE}

Al-Jukr M., 2009. Al-kurat dakhalt dubaî 'âm 1928 biwâstah « al-'ulâmah muhamad nûr ». AlBayan.

Amara M., 2012. Sports, Politics and Society in the Arab World. Londres, Palgrave Macmillan, 192 p.

Amara M., 2019. Tennis in the Middle East and North Africa. In Lake R. J., Osborne C. A. (dir.), Handbook of Tennis. Routledge, Londres, chapitre XV.

Chicchi F., Simone A., 2017. La societtà della prestazione. Rome, Ediesse, 211 p. 
Davidson C., 2008. Dubaï the vulnerability of the success. Londres, Hurst Publishers, $392 \mathrm{p}$.

Davis U., 2000. Conception of citizenship in the Middle East: State, Nation and People. In Butenschøn N. A., Davis U., Hassassian M. (dir.), Citizenship and the State in the Middle East. NewYork, Syracuse University Press, p. 49-69.

Dumortier B., Lavergne M., 2002. Doubaï: ville du pétrole ou projet métropolitain post-pétrolier. Annales de Géographie, $\mathrm{n}^{\circ}$ 623, 2002, p. 41-59.

Dumortier B., 2007. Développement économique et contournement du droit: les zones franches de la rive arabe du golfe Persique. Annales de Géographie, $\mathrm{n}^{\circ}$ 658, p. 628-644.

Elsheshtawy Y., 2009. Dubaï an Urban Spectacle. Londres, Routledge, 305 p.

Elsheshtawy Y., 2019. Temporary Cities: Resisting Transience in Arabia. Londres, Routledge, 312 p.

Herb M., 2014. The Wage of Oil: Parliaments and Economic Development in Kuwait and the UAE. Ithaca et Londres, Cornell University Press, $258 \mathrm{p}$.

Illouz E., Cabanas E., 2018. Happycratie. Comment l'industrie du bonheur a pris le contrôle de nos vies? Paris, Premier Parrallèle, 300 p.

Kanna A., 2011. Dubaï: The City as Corporation. Minneapolis, University of Minnesota Press, 296 p.

Kapiszewski A., 2006. Arab versus Asian Migrant Workers in the GCC Countries. Travail présenté dans le cadre de l'United Nations Expert Group Meeting on International Migration and Development in the Arab Region, Population Division, Department of Social and Economic Affairs, United Nations Secretariat, Beirut.

Kazerouni A., 2017. Le miroir des Cheikhs. Musées et politique dans les principautés du golfe Persique. Paris, PUF, $272 \mathrm{p}$.

Khalaf A., AlShehabi O., Hanieh A. (dir.), 2015. Transit States. Labour, Migration and Citizenship in the Gulf. Londres, Pluto Press, 266 p.

Khalaf S., 2000. Poetics and politics of newly invented traditions in the Gulf: Camel racing in UAE. Ethnology, vol. 39 n 3, p. 243-261.

Khalaf S., 2010. Dubaï Camel Market Transnational Workers: An Ethnographic Portait. City and Society, vol. 22, p. 97-118.

Kinninmont J., 2013. Citizenship in the Gulf. In Echagüe A. (dir.), The Gulf States and the Arab Uprisings. Espagne, FRIDE and Gulf Research Center, p. 47-58.

International Labour Organisation, 2009. International Labour Migration and Employment in the Arab Region. Arab Employment Forum.

Lavergne M., 2002. Dubaï ou la métropolisation incomplète d'un pôle de l'économie monde. Cahiers de la Méditerranée, ${ }^{\circ}$ 64, p. 257-296.

Lavergne M., 2009. Dubaï, utile ou futile. Hérodote, n 33, p. 32-57.

Lussault M., 2010. L'urbain métropolisé en voie de généralisation. Constructif, n²6. URL: http:// www.constructif.fr/bibliotheque/2010-6/l-urbain-metropolise-en-voie-de-generalisation.html? item_id=3029

Le Magoariec R., 2019. Sport et images... ou la mise en scène des monarchies du Golfe. MoyenOrient, $\mathrm{n}^{\circ} 43$, p. 68-73. 
Le Magoariec R., 2019. Sport dans le Golfe. La naturalisation, une arme pour gagner. Orient XXI. URL: https://orientxxi.info/magazine/sport-dans-le-golfe-la-naturalisation-une-arme-pourgagner,3052

Le Magoariec R., 2020. Le cricket, improbable passion dans le Golfe. Orient XXI. URL: https:// orientxxi.info/magazine/le-cricket-improbable-passion-dans-le-golfe,3670

Le Magoariec R., 2020. Les pays du Golfe très à cheval sur les courses de chameaux. Orient XXI. URL: https://orientxxi.info/magazine/les-pays-du-golfe-tres-a-cheval-sur-les-courses-dechameaux,4010

Le Renard A., 2019. Le privilège occidental : Travail, intimité et hiérarchies postcoloniales à Dubaï. Paris, Les Presse de Science Po, $272 \mathrm{p}$.

Marchal R. (dir .), 2001. Doubaï: Cité globale. Paris, CNRS Éditions, 131 p.

Moghadam A., 2013. L'Iran recomposé de Dubaï. Étude des pratiques et discours des migrants iraniens. Thèse de doctorat en géographie, aménagement et urbanisme, sous la direction de Marc Lavergne, Lyon, université de Lyon 2.

Montagne C., 2013. Le renforcement du modèle de développement urbain de Dubaï après la crise. Urbanités, 08/11/2013. URL: https://www.revue-urbanites.fr/le-renforcement-du-modele-dedeveloppement-urbain-de-dubai-apres-la-crise/

Montagne C., 2016. Développement urbain et planification urbaine à Abu Dhabi et à Dubaï. Politiques, acteurs et mobilité. Thèse de doctorat en aménagement, urbanisme et dynamiques des espaces, sous la direction de Guy Chemla, Paris, université de Sorbonne Paris 4.

Morgan R., 2007. The Encyclopaedia of World Cricket. York, SportsBook Ltd, 344 p.

Queval I., 2004. S'accomplir ou se dépasser. Essai sur le sport contemporain. Paris, Gallimard, 352 p.

Stadnicki R., 2019. Les revers de l'image: la publicité urbanistique dans le Golf. Revue Marketing Territorial, $\mathrm{n}^{\circ} 2$.

Ulrichsen Coates K., 2016. The UAE: Power, Politics and Policy making. Londres, Routledge, 270 p.

Vigarello G., 2012. Le défi actuel de l'apparence. Une tragédie? Communications, n 91, p 191-200.

Vora N., 2013. Impossible Citizens. Dubai’'s Indian Diaspora. Londres \& Durham, Duke University Press, $246 \mathrm{p}$.

\section{NOTES}

1. Par l'expression «sport spectacle » nous entendons la mise en scène du sport dans le but de créer une industrie de divertissement.

2. Cette phase migratoire se matérialise surtout par un renforcement au sein des différentes strates sociales des Émirats arabes unis de la présence asiatique. En 2009, d'après les chiffres de l'Organisation Internationale du Travail et du Forum arabe pour l'emploi, parmi les travailleurs étrangers présents aux Émirats arabes unis $87 \%$ sont issus du continent asiatique, quand les Américains et Européens, qui occupent des fonctions à responsabilités, ne représentent que $3 \%$ de ces travailleurs. Sur la période 2002-2004, d'après les sources de l'universitaire Andrezj Kapiszewski, aux EAU, les Indiens et Pakistanais, avec respectivement, 1,2 millions d'habitants et 450000 habitants sont les communautés les plus représentées sur le territoire émirien. Une large partie de ces habitants se concentrent autour de la conurbation que constituent Dubaï, Sharjah et Ajman. 
3. Depuis mai 2017, les clubs d'Al-Shebab et d'Al-Ahly ont été fusionnés sous la direction de l'émir Mohammed bin Rachid Al-Maktoum, une fusion qui s'explique en partie par les problèmes financiers que rencontre le sport émirien.

4. Source : entretien avec Walid Al-Hosani, directeur général de l'Arabian Gulf League, Abu Dhabi, 2018.

5. Source : entretien avec Ali Omar Al-Balouchi, directeur du développement sportif au Dubaï Sports Council, Dubaï, 2018.

6. Source : entretien personnel avec un responsable de la formation travaillant pour le Dubaï Sports Council, Dubaï, 2018.

7. Le recensement de 2018 comptabilise une population de 9,5 millions de résidents vivant aux Émirats arabes unis. Pour des questions d'ordre politique ce recensement ne donne pas de chiffres par nationalité, c'est donc en compilant les chiffres fournis par les différentes ambassades étrangères en poste aux Émirats arabes unis que l'on obtient cette estimation de $11,4 \%$.

8. Dubaï Statistics Center, Yearly population estimates, 2018.

9. Source: entretien personnel avec un responsable de la formation travaillant pour le Dubai Sports Council, Dubaï, 2018.

10. Source : entretiens personnels avec deux responsables impliqués dans le développement de l'éducation physique à l'échelle d'Abu Dhabi, Abu Dhabi, 2018.

11. Source : entretien personnel avec un sportif de haut niveau émirien, Abu Dhabi, 2018.

12. Source : entretien avec un acteur travaillant dans le domaine de la voile aux Émirats arabes unis, Dubaï, 2018. Une tendance confirmée à de multiples reprises lors d'entretiens avec un sportif émirien de haut niveau, avec un homme d'affaires travaillant dans le football émirien et deux membres du Dubaï Sports Council : Ali Omar Al-Balouchi directeur du développement sportif et un responsable de la mise en place des programmes de formation sportive. Abu Dhabi, 2018, Dubaï, 2018.

13. L'équivalent d'une somme de 1000 euros.

14. Source : entretiens avec un sportif émirien de haut niveau, un formateur travaillant pour le Dubaï Sports Council et un homme d'affaires évoluant dans les coulisses du football golfien.

15. Source: entretien personnel avec Ali Khaled, journaliste et réalisateur du documentaire Anwâr Rômâ sur la qualification de la sélection émirienne, en 1990, pour sa première et unique participation à la Coupe du monde de football, Dubaï, 2018.

16. Source : entretien avec Walid Al-Hosani, directeur général de l'Arabian Gulf League, Abu Dhabi, 2018.

17. Source: entretiens avec trois spécialistes travaillant dans le football à Dubaï et un responsable de la mise en place des programmes de formation du Dubaï Sports Council, Dubaï, 2018.

18. D'après la loi fédérale $n^{\circ} 6$, ce système de conscription obligatoire oblige les hommes de 18 à 30 ans à réaliser un service militaire allant de 9 mois à 2 ans en fonction de leur niveau d'études, tandis que pour les femmes cet engagement reste facultatif. Seuls les hommes étant responsables financiers et légaux de leur famille sont exemptés de participer au service national. Au terme de leur formation militaire, les hommes et femmes rejoignent la réserve nationale. Le Premier ministre des EAU et émir de Dubaï, Mohammed Bin Rachid Al-Maktoum, présente ce système de conscription obligatoire comme un vecteur participant à renforcer la protection de la nation et enseigner aux jeunes les valeurs de la loyauté, de discipline et de sacrifice. Sources: Palais présidentiel d'Abu Dhabi, 28/06/2014, " qânûn îtihâdî raqam (6) lisanat 2014 fî chân al-khidmat alwatanîat walâihtîâtîa ", șadar 'inâ fî qasr alr'îyasat bi âbûzabi (Publication du palais présidentiel). $\mathrm{BBC}$ version arabe, 20/01/2014. "al'îmârât ta'etazim tatbîq nizâm alkhidmat al'askarîat al'iilzâmia ", BBC, <al'iimarat taetazim tatbiq nizam alkhidmat aleaskariat al'iilzamia>.

19. Source : entretien personnel avec un journaliste travaillant à Dubaï, Dubaï, 2018. 
20. Trucial States ou États de la Trêve, cet ensemble géographique comprenaient onze émirats - les sept émirats actuels de la fédération des Émirats arabes unis plus quatre autres ayant ensuite été absorbés par les émirats de Fujairah et Sharjah- ces émirats à l'origine de la future fédération des EAU tirent leur nom d'accords passés avec les Britanniques au cours de la seconde moitié du XIXe siècle. Par ces différents accords, les Britanniques marquent alors la volonté de lutter contre la piraterie dans la région après avoir, en 1820, coulé la flotte des Al-Qawasim; par ailleurs, pour les Britanniques, ces traités visent à étendre leur influence sur les rives du Golfe arabo-perisque. En 1971, les États de la Trêve prennent le nom d'Émirats arabes unis, Ras Al-Khaimah, le dernier émirat appartenant à cet ensemble géographique, rejoint en dernier la fédération, en 1972.

21. Source : entretien avec le journaliste Paul Radley, spécialiste de cricket, Abu Dhabi, 2018.

22. Source: entretien personnel avec le journaliste Paul Radley, Abu Dhabi, 2018, et un haut responsable du cricket de Dubaï, Dubaï, 2018.

23. Source : entretien personnel avec le journaliste Paul Radley, Abu Dhabi, 2018, et un haut responsable du cricket de Dubaï, Dubaï, 2018.

24. Source : entretien avec un haut responsable du cricket de Dubaï, Dubaï, 2018.

25. 1000 AED : 232 euros; 3000 AED : 698 euros.

26. 650 AED : 151 euros.

27. Entretien avec un haut responsable du cricket de Dubaï, Dubaï, 2018.

28. Entretien personnel avec Iqbal Husseïn Chaudhry, Doha, 2021.

29. Source : entretiens personnels auprès de plusieurs joueurs de tape ball cricket (Ali, Eldho, Muhammad, Talha, Hamza), Dubaï, 2018.

30. Source : entretien personnel avec Tony Robinson coach de l'équipe de football américain des Abu Dhabi Wildcast, Abu Dhabi, 2018.

31. Source : entretien personnel avec Jennifer Luckhurst et Durant Gerber, managers du club Dubaï Exiles, Dubaï, 2018.

32. Source : entretiens personnels avec une émirienne, Roubah, et trois femmes expatriées vivant à Dubaï (Fatima, Julia et Samantha), Dubaï, 2018.

33. Source : entretien avec un agent de joueur de football, Dubaï, 2018.

34. Source : entretiens personnels avec un agent de joueur, un responsable du développement de la formation au Dubaï Sports Council et un journaliste qui suit le championnat de football émirien, Dubaï, 2018.

35. Source : entretiens personnels avec un agent de joueur, un responsable du développement de la formation au Dubaï Sports Council et un journaliste qui suit le championnat de football émirien, Dubaï, 2018.

36. Pour aller plus loin sur ce sujet se reporter aux thèses respectives de Clémence Montagne (2016) et Amin Moghadam (2013).

37. World Health Organization, Diabetes country profiles, 2016. URL: https://www.who.int/ diabetes/country-profiles/are_en.pdf

\section{RÉSUMÉS}

L'émirat de Dubaï demeure l'une des premières principautés du Golfe à avoir investi la sphère du sport spectacle au cours de la décennie 1990. Témoin des changements de génération au sein du pouvoir dubaïote, l'intégration à ce domaine marque aussi l'entrée de l'émirat dans une nouvelle 
ère par une image nouvelle de « cité globale ». Étudier Dubaï à travers le prisme du sport permet de comprendre, d'une part, comment l'émirat investit le terrain sportif et quels sont ses objectifs affichés, d'autre part, comment le sport se vit et se pratique au sein de la société aux origines multiples évoluant dans l'émirat. Le sport permet ainsi de mettre au jour, non pas un, mais plusieurs visages de Dubaï qui se superposent les uns aux autres sans jamais réellement se côtoyer sur le terrain sportif.

The emirate of Dubaï remains one of the first emirates to have invested in the sport industry during the 1990s. Witnessing the changes of generation within the power of Dubai, integration in this domain also marks the entry of the emirate into a new era by the building of a new image of "global city ». Studying Dubaï through the prism of sport allows us to understand, on the one hand, how the Emirate invests in the field of sport and what its stated goals are, on the other hand, how sport is experienced and practiced in this multi-ethnic society evolving in the emirate. Thus sport brings us a look at, not one, but several Dubaï faces that are superimposed on each other without ever too much rubbing shoulders on the sports ground.

\section{INDEX}

Keywords : Dubaï, United Arab Emirates, sport, geopolitics, segregation, stadium, image, expatriate

Thèmes : Sur le Champ

Mots-clés : Dubaï, Émirats arabes unis, sport, géopolitique, ségrégation, stade, image, expatrié

\section{AUTEUR}

\section{RAPHAËL LE MAGOARIEC}

Raphaël Le Magoariec, rlemagoariec@hotmail.fr, est chercheur-doctorant au sein de l'Équipe Monde Arabe et Méditerranée, laboratoire CITERES de l'université de Tours. Il a récemment publié :

- Le Magoariec R., 2021. PSG-Manchester City: si loin du Golfe, si proche du Golfe. The Conversation [En ligne], 27/09/21, URL: https://theconversation.com/psg-manchester-city-si-loin-du-golfe-siproche-du-golfe-168728?utm_source=linkedin\&utm_medium=bylinelinkedinbutton - Le Magoariec R., 2021. La difficile trajectoire du sport yéménite. ID4D [En ligne], 22/02/2021. URL: https://ideas4development.org/difficile-trajectoire-sport-yemenite/ - Le Magoariec R., 2020. Les stades de la Coupe du monde 2022, reflets d'un Qatar à deux vitesses. Les Cahiers d'EMAM, $\mathrm{n}^{\circ} 33$. 